\title{
The study of water capacitance for single electric cable in precision measurement
}

\author{
Jian-Kun Tu ${ }^{\mathrm{a}}$, Hai-Ma Yang ${ }^{\dagger, a, b}$, Bin Jiang ${ }^{\mathrm{a}}$ and Jin $\mathrm{Liu}^{\mathrm{c}}$ \\ ${ }^{a}$ Shanghai Electric Cable Research Institute, \\ Shanghai, 200093, China \\ ${ }^{b}$ University of Shanghai for Science and Technology, \\ Shanghai, 200093, China \\ 'Shanghai University of Engineering and Science, \\ Shanghai, 201620,China \\ tE-mail: snowyhm@sina.com \\ www.secri.com
}

\begin{abstract}
A high precision water capacitance measuring system is designed by using a water capacitive sensor, which carries on non-contact measurement in a single electric cable in water. System's fundamental and electric data processing methods have been illustrated through the analysis of object's features. The factor of influenced sensor's measurement accuracy and stability is analyzed as well. Then the paper proposes a technical means to utilizing electronic and digital signal filtering technology for enhancing accuracy of sensors. It provides a means of measuring the quality of high speed data communication cables. The experimental data analysis shows that the measurement range of the system is $10-300 \mathrm{pF}$, the resolution is better than $0.2 \mathrm{pF}$, which can meet the needs of the water capacitance detection in the production of insulated wire electric cable.

Keywords: Water Capacitance; Single Electric Cable; Precision Measurement; Electric Bridge.
\end{abstract}

\section{Introduction}

Among all the parameters of the electric cable, the water capacitance is the most representative parameter, which can synthesize the heterogeneity of the core line. The change of capacitance in water can reflect the process parameters of electric cable production process changes and changes in environmental conditions. By detecting changes in cable capacitance in water, the electric cable production conditions can be controlled well. So water capacitance testing for single electric cable is very important significance in the production of the electric cable [1].

In this paper, a particular single electric cable in water is used as the main research object, and a measuring system is built by using National Instrument (NI) data acquisition board and Personal Computer (PC) [2]. A high accuracy capacitive sensor is applied to the single electric cable to measure the various 
changes. Moreover, temperature sensors are also applied to the water tank. Then, the relation curve between the temperature and capacitance is obtained.

\section{The Design of Measuring System}

The measurement system is consisted by the following: single electric cable in water, high precision capacitive sensor, NI multichannel data collection board, PC and other accessory device and testing software et al. The multichannel data collection instrument is based on high performance of Digital Signal Processing (DSP) processor and computer technology. With the support of low-level control procedure and data acquisition software, it accomplishes collection, processing, and display of the water copacitance data. The whole system organization is illustrated in Fig.1.

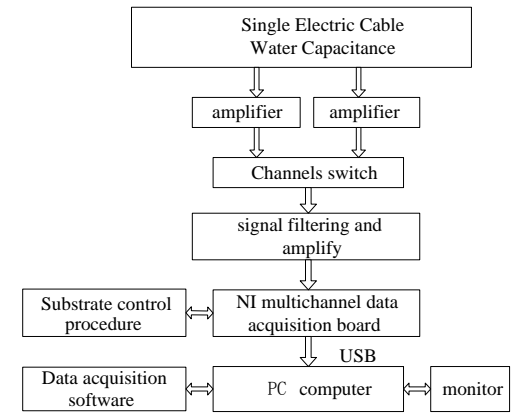

Fig. 1. The structure chart of measurement system

And the test equipment installation is shown in Fig. $2^{[3]}$.

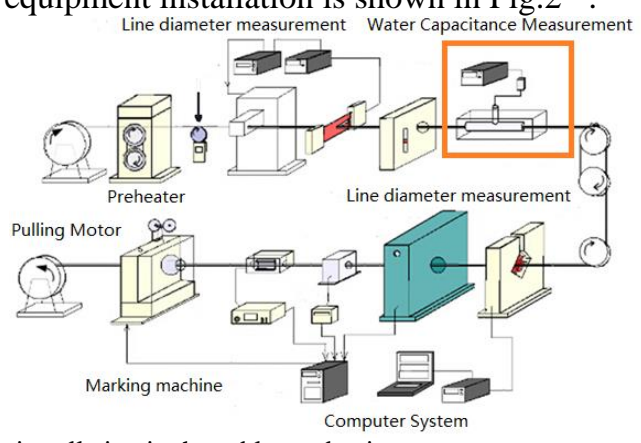

Fig.2. Test equipment installation in the cable production

\section{The Optimization Design of Capacitance Sensing Circuit}

Water capacitance sensor is commonly used at tiny capacitance measurement [45]. Because of temperature, noise and null drift of capacity sensor, a greater measuring error will be led. Simultaneously, bridge form is adopted commonly 
at the measurement of the circuit layout. The amplified power supply error has an influence on the output signal. Therefore overcoming these two influencing factors is the key point to improve the accuracy of capacitive sensor. This paper will analysis and reduce these two source error in two aspects. The whole measuring circuit functional scheme is shown in Fig.3.

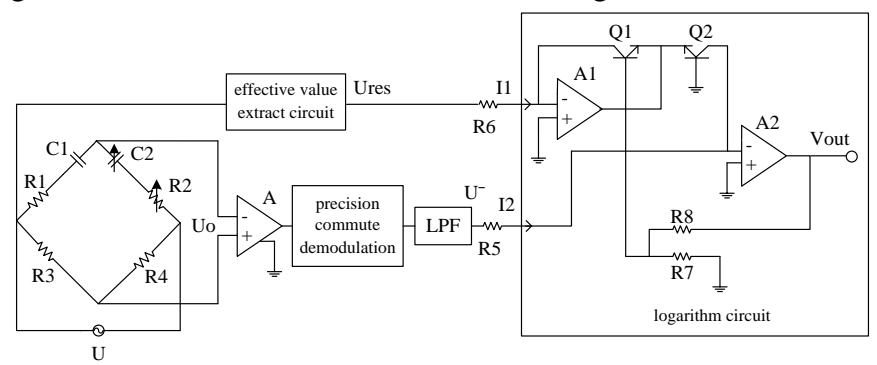

Fig. 3. The schematic of capacitance sensing circuit

\section{1 .The measuring principle of capacitive sensor}

The principle of capacitive measurement is based on the principle of the parallel plate capacitor. For conductive targets, the sensor and the target opposite form the two plate electrodes. If neglect the fringe effect of capacitive sensor, the measurement capacitance $\mathrm{C}$ can be expressed by the following simple equation.

$$
\mathrm{C}=\frac{\varepsilon_{r} \varepsilon_{0} S}{\delta}=\frac{\varepsilon S}{\delta}
$$

Where $S$ is the area of electrode, $\delta$ is the distance between the two electrodes, $\varepsilon_{r}$ is the relative permittivity, $\varepsilon_{0}$ is the permittivity of vacuum, and $\varepsilon$ is the dielectric constant.

The dielectric constant between the electrodes changes when the material is not the same, and then the capacitance changes as well. The capacitance change $\Delta \varepsilon$ is presented as.

$$
\Delta \mathrm{C}=C_{o} \Delta \varepsilon
$$

Where $C_{o}$ is the initial capacity, when the electrode distance was $\delta$.

Since the changes of capacitance change are very small, the dedicated measuring circuit is needed to set up to get proportional voltage signal to the capacitance change. Capacitive sensor switches in an arm of the alternating current bridge, while the other arm is steady resistance and capacitance. The remaining arms are constant resistance. The alternating current bridge is excited by constant amplitude sinusoid, $U=U_{\mathrm{m}} * \sin \omega$ t as carrier wave, which drives the bridge circuit.

The four arms of bridge impedance are 


$$
\mathrm{Z}_{1}=R_{1}-j \frac{1}{\omega C_{1}}, Z_{2}=R_{2}-j \frac{1}{\omega C_{2}}, Z_{3}=R_{3}, Z_{4}=R_{4}
$$

, and the output voltage is,

$$
\Delta U o=\mathrm{U} \frac{Z_{1} Z_{4}-Z_{2} Z_{3}}{\left(Z_{1}+Z_{2}\right)\left(Z_{3}+Z_{4}\right)}
$$

The initial alternating current bridge is assumed to be balanced. When the working capacitance $\mathrm{C} 1$ changes $\Delta \mathrm{C}$, it makes impedance $\mathrm{Z} 1$ changes $\Delta \mathrm{Z} 1$. The output voltage $\Delta U o$ can be written,

$$
\Delta U o=\mathrm{U} \frac{\frac{Z_{4} \Delta Z_{1}}{Z_{3} Z_{1}}}{\left(1+\frac{Z_{2}}{Z_{1}}+\frac{\Delta Z_{1}}{Z_{1}}\right)\left(1+\frac{Z_{4}}{Z_{3}}\right)}
$$

In respect that the initial symmetry bridge is balanced, the denominator of $\Delta Z_{1}$ item is the factor caused by nonlinear. Considering its value is relatively small that can be omitted, the linear output can be described as following equations:

$$
\begin{gathered}
U o=\frac{U}{4} \frac{\Delta Z_{1}}{Z_{1}} \\
\Delta Z_{1}=\frac{\Delta C}{j w C o(C o+\Delta C)}
\end{gathered}
$$

The initial values of parameters are

$$
C_{1}=C_{2}=1 n F, R_{1}=R_{2}=R_{3}=R_{4}=300 \Omega \text {. }
$$

Inserting the values of parameters into (5) and (6), the relationship between output voltage $U o$ and capacitance changes $\Delta C$ can be obtained, as shown in the following table 1 . The measuring range of $\Delta C$ is $0-300 \mathrm{pF}$

Table 1. The relationship between the output voltage $U o$ and capacitance variation $\Delta C$

\begin{tabular}{|l|c|c|c|c|c|c|c|c|c|c|}
\hline \hline $\begin{array}{c}\Delta C \\
(\mathrm{pF})\end{array}$ & 10 & 20 & 30 & 40 & 50 & 100 & 150 & 200 & 250 & 300 \\
\hline \hline $\begin{array}{l}U O \\
(\mathrm{mV})\end{array}$ & 1.9 & 3.7 & 4.5 & 5.3 & 6.2 & 10.9 & 14.8 & 18.6 & 22.5 & 30.3 \\
\hline \hline
\end{tabular}

\subsection{The method of reduce measurement error}


The capacitive sensor circuit has two main errors. The first one is from voltage supplying, and another is thermal noise which is from bridge circuit. Reducing the impact of these two errors is the key point to improve the capacitive bridge precision.

The method of reducing the error of voltage supply can be solved by the logarithmic circuit. The principle of circuit is shown in Fig.4.

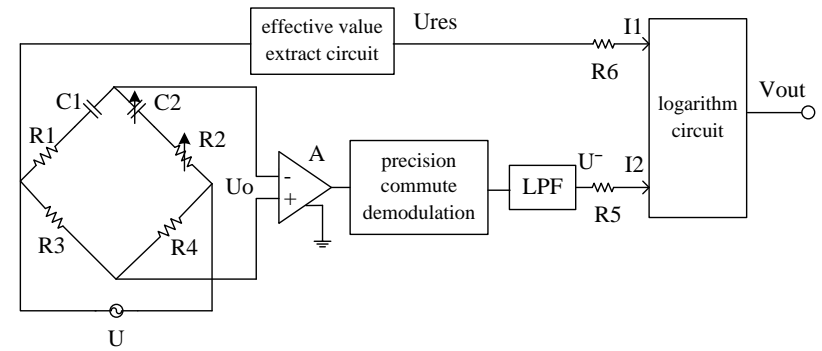

Fig. 4. Eliminate the error of voltage supply in capacitance sensing circuit

Assuming the offset voltage of voltage supply is $\sigma$, and then the power supply $\mathrm{U}$ can be described as,$U=\left(U_{m}+\sigma\right) * \sin \omega \mathrm{t}$.

Through the effective value extract circuit,

$$
U_{r e s}=\sqrt{\frac{1}{T} \int_{0}^{T}\left(U_{m} \sin \omega t\right)^{2} d t}=\frac{U_{m}}{\sqrt{2}}
$$

The output voltage of logarithmic circuit is,

$$
V_{\text {out }}=L \log \frac{I_{1}}{I_{2}}=L\left(\log \frac{2 \pi}{P A v}+\log \frac{\beta}{\alpha}-\log \varepsilon\right)
$$

The $\mathrm{L}$ is constant. And the letter $\alpha$ and $\beta$ are the top and bottom boundary of the input current value of $\mathrm{I}_{2}$ and $\mathrm{I}_{1}$ in logarithmic circuit.

According to the above equations, the output signal of bridge through the logarithmic operation can be acquired from the uniform function relationship between the output function and changes in capacitive.

\section{The Result of Test Measurement}

After the original electric bridge data is collected by DAQ board, a curve can be got just like Fig.5.

Through the test data, water capacitance value from $10 \mathrm{pF}-300 \mathrm{pF}$ can be measured by this bridge circuit. Under the data processing and analysis, the resolution of system comes up to $0.2 \mathrm{pF}$, and linearity is superior to $0.1 \mathrm{mV} / \mathrm{pF}$.. 


\section{Conclusions}

According to the analyses of source noise in the capacitance sensor, the measuring circuit, the data processing means are improved, and the precision is raised as well. The capacitive sensing circuit has a non-linear error. In order to reduce the non-linear error exploiting the differential capacitive sensors is the best choice.

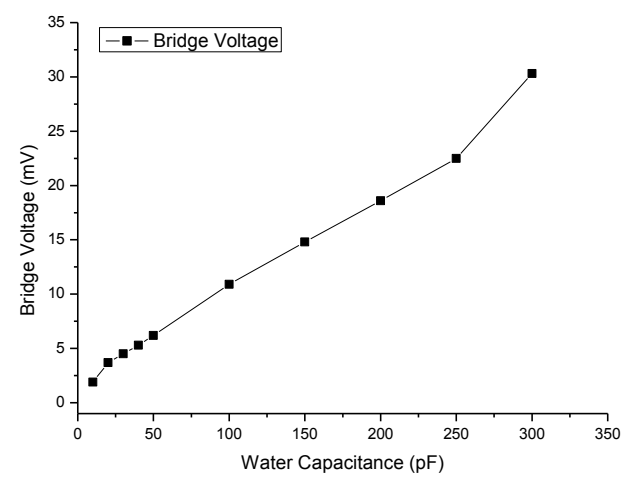

Fig.5. Curve of original electric bridge data

After all, the measurement system is designed by high accuracy capacitive sensor. And through using the logarithmic circuit, it satisfied the requirements of single electric cable production, and has a great reference for the capacitive sensor in the similar non-contact, high-precision applications.

\section{Acknowledgement}

This work is supported by the Natural Science Foundation of Shanghai under Grants 15ZR1417400. Work partially supported by the National Natural Science Foundation of China under Grant 61302181.

\section{References}

1. Lou Li-min, Wang Zhaojun, Mao Shumin et al. The Research on Calibration Method of The Capacitance Measurement Systems [J]. Metrology \& Measurement Technique, 2012, 39(2):3-5.

2. Xu Xinghua. Online testing of capacitance in water in communication cable [J]. Journal of Ningbo Polytechnic,2007,11(5): 78-80.

3. Lin Chunyao, Yang Xian, Ma Zhiqin et al. Influence of moisture and aging on dielectric property of oil-paper in condenser bushing [J]. Southern Power System Technology, 2016,10(1):18-25. 
4. Li Min, Zhao Hong, Jiang Hua. Online prediction of SRL by FFT of cable capacitance in water[J]. Wire \& Cable,2004,(2):17-22.

5. Harry Prunk. The latest development in measuring equipment for online quality control at the cable extrusion[J]. Wire Journal International 2000. 